\title{
Ação da Lippia sidoides Cham nas infecções de faringe, cavidade oral e problemas odontológicos: uma revisão de literatura
}

Action of Lippia sidoides Cham on infections of the pharynx, oral cavity and dental problems: a

\author{
literature review
}

Acción de Lippia sidoides Cham sobre infecciones de faringe, cavidad oral y problemas dentales: revisión de la literatura

\section{Resumo}

O objetivo desse estudo foi realizar uma revisão da literatura sobre o uso da planta Lippia sidoides Cham no tratamento de infecções de faringe e cavidade oral. Dessa forma, realizou-se uma pesquisa da literatura a partir de artigos indexados em bibliotecas virtuais internacionais, Scientific Eletronic Library Online (SciELO), U.S. National Library of Medicine (PubMed), Google Acadêmico e Portal Periódicos Capes. Os descritores utilizados foram: Lippia sidoides Cham, infecções de faringe e cavidade oral, e seus correspondentes em inglês. A Lippia sidoides Cham pertence à família verbenaceae, e é conhecida popularmente como alecrim-pimenta. Na medicina popular, essa planta é usada principalmente na forma de infusão para o tratamento de infecções da boca e da garganta. As propriedades terapêuticas dessa planta estão relacionadas aos constituintes majoritários o carvacrol e o timol, uma vez que essas moléculas possuem ação anti-inflamatória, antioxidante, antifúngica e antibacteriana. Os estudos comprovam a propriedade antibacteriana e antifúngica dos extratos e do óleo essencial dessa planta frente às cepas de bactérias cariogênicas, como Streptococcus mutans e frente as cepas de diferentes espécies de Candida, que é o principal fungo relacionado as infecções da cavidade oral. Porém, apesar da Lippia sidoides Cham ser usada na medicina tradicional para o tratamento de infecções relacionadas a faringe faltam dados e estudos clínicos que sustentem essa prática. Assim, conclui-se que o extrato da planta Lippia sidoides Cham é bastante utilizado popularmente e comercializado para infecções relacionadas a faringe, cavidade oral e problemas odontológicos.

Palavras-chave: Lippia sidoides Cham; Infecção de faringe; Infecção de cavidade oral.

\section{Abstract}

The aim of this study was to review the literature on the use of the plant Lippia sidoides Cham in the treatment of pharyngeal and oral cavity infections. Thus, a literature search was carried out based on articles indexed in 
international virtual libraries, Scientific Electronic Library Online (SciELO), U.S. National Library of Medicine (PubMed), Academic Google and Capes Journal Portal. The descriptors used were: Lippia sidoides Cham, infections of the pharynx and oral cavity, and their English counterparts. Lippia sidoides Cham belongs to the verbenaceae family and is popularly known as pepper rosemary. In folk medicine, this plant is mainly used as an infusion to treat infections of the mouth and throat. The therapeutic properties of this plant are related to the major constituents carvacrol and thymol, since these molecules have anti-inflammatory, antioxidant, antifungal and antibacterial action. The studies prove the antibacterial and antifungal properties of the extracts and essential oil of this plant against cariogenic bacteria strains, such as Streptococcus mutans and against strains of different Candida species, which is the main fungus related to infections in the oral cavity. However, although Lippia sidoides Cham is used in traditional medicine for the treatment of infections related to the pharynx, data and clinical studies to support this practice are lacking. Thus, it is concluded that the extract of the plant Lippia sidoides Cham is widely used and marketed for infections related to the pharynx, oral cavity and dental problems.

Keywords: Lippia sidoides Cham; Pharyngeal infection; Oral cavity infection.

\section{Resumen}

El objetivo de este estudio fue revisar la literatura sobre el uso de la planta Lippia sidoides Cham en el tratamiento de infecciones faríngeas y de la cavidad oral. Así, se realizó una búsqueda bibliográfica basada en artículos indexados en bibliotecas virtuales internacionales, Scientific Electronic Library Online (SciELO), U.S. National Library of Medicine (PubMed), Academic Google y Capes Journal Portal. Los descriptores utilizados fueron: Lippia sidoides Cham, infecciones de faringe y cavidad oral y sus contrapartes en inglés. Lippia sidoides Cham pertenece a la familia de las verbenaceae, y es conocida popularmente como pimienta romero. En la medicina popular, esta planta se utiliza principalmente como infusión para tratar infecciones de la boca y la garganta. Las propiedades terapéuticas de esta planta están relacionadas con los principales constituyentes carvacrol y timol, ya que estas moléculas tienen acción antiinflamatoria, antioxidante, antifúngica y antibacteriana. Los estudios demuestran las propiedades antibacterianas y antifúngicas de los extractos y aceite esencial de esta planta frente a cepas de bacterias cariogénicas, como Streptococcus mutans, y frente a cepas de diferentes especies de Candida, principal hongo relacionado con las infecciones de la cavidad bucal. Sin embargo, aunque Lippia sidoides Cham se utiliza en la medicina tradicional para el tratamiento de infecciones relacionadas con la faringe, faltan datos y estudios clínicos que apoyen esta práctica. Así, se concluye que el extracto de la planta Lippia sidoides Cham es ampliamente utilizado y comercializado para infecciones relacionadas con la faringe, cavidad bucal y problemas dentales.

Palabras clave: Lippia sidoides Cham; Infección faríngea; Infección de la cavidad oral.

\section{Introdução}

O uso das plantas medicinais para o tratamento e/ou cura de doenças surgiu com as descobertas das propriedades medicamentosas existentes em alguns vegetais. Desde a antiguidade essa prática está presente na humanidade e continua sendo utilizada na atualidade por meios dos ensinamentos que são transmitidos ao longo das gerações (Silva;, Vieira, \& Gurgel, 2017).

O Brasil é o país com a maior biodiversidade do planeta, cerca de $15 \%$ a $20 \%$ da totalidade mundial. Dentre os elementos que constituem essa biodiversidade estão as plantas, que são a matéria-prima para a produção de fitoterápicos. Além disso, o Brasil possui um grande potencial genético para o avanço de novos medicamentos e, quando associada à rica diversidade étnica e cultural detém um importante conhecimento tradicional relacionado ao uso de plantas medicinas (Brasil, 2009; Martins et al., 2020).

Além disso, percebendo que essa cultura está muito presente na sociedade brasileira e analisando a importância e funcionalidade dessas plantas, em 2007, foram realizadas as primeiras implantações de medicamentos fitoterápicos no elenco de referência de medicamentos e insumos complementares para a assistência farmacêutica na atenção básica em saúde, de acordo com a portaria MS/GM n. 3.237.24 (Lima \& Gomes, 2014).

No ano de 2012, de acordo com a portaria do Ministério de Saúde n.533, a lista de medicamentos de referências do SUS, a Relação Nacional de Medicamentos Essenciais (RENAME), introduziu pela primeira vez 12 fitoterápicos padronizados no âmbito do Sistema Único de Saúde (SUS) (Mendonça et al., 2018). No advento do terceiro milênio, aproximadamente 3/4 da população mundial dependem ainda do potencial medicinal das plantas. Além disso, muitas vezes essas plantas são utilizadas 
como o único recurso medicamentoso de uma grande parcela da população (M. D. M. M. Gonçalves et al., 2018; Madaleno, 2011).

A flora é considerada uma fonte muito importante de medicamentos para a melhoria da saúde e tratamento de várias doenças em diversas comunidades humanas. Por isso, nos últimos anos, a demanda por medicamentos fitoterapêuticos e outros produtos derivados de plantas de várias espécies vêm aumentando de modo significativo (Mustafa et al., 2017). Plantas medicinais são consideradas fontes para novos fármacos, com ação antisséptica, antifúngica e antibacteriana. Além de muitas vezes serem mais acessíveis para pacientes, uma vez que podem possuir menor custo (Faria \& Iecher, 2014). Algumas espécies de plantas do gênero Lippia, dentre elas a espécie, Lippia sidoides Cham, há muito tempo vêm sendo testadas para o tratamento de diversas doenças do homem, devido ao potencial bioativo presente nesse gênero (Soares \& Tavares-Dias, 2013).

Lippia sidoides Cham pertence à família verbenaceae, é conhecida popularmente como alecrim-pimenta, alecrim, alecrim-bravo entre outros a depender da região do Brasil e outros países. Essa planta tem maior ocorrência na região nordeste do Brasil, podendo ser encontrada, também em outros países da América Latina (Saúde, 2018). Essa planta possui porte ereto, arbustivo com muitas ramificações, um caule grosso, podendo atingir até três metros de altura, folhas aromáticas e flores diminutas e esbranquiçadas. Suas propriedades anti-inflamatória, antimicrobiana e antifúngica já foram descritas na literatura (de Morais et al., 2016; Souza et al., 2020).

Essa planta é considerada como uma das espécies vegetais com potencial para a geração de produtos e faz parte da Lista Nacional de Plantas Medicinais de interesse para o Sistema Único de Saúde (RENISUS). As folhas da Lippia sidoides Cham, na forma de infusão, são utilizadas na medicina tradicional como alternativa para o tratamento de infecções bucais e da garganta (Brasil, 2009; Souza et al., 2020).

As infecções bucais são consideradas como um dos grandes problemas de saúde pública, devido à alta incidência e predominância. Estudos demonstram que existe uma associação importante entre o estado de saúde geral e o estado da saúde bucal, ou seja, uma relação entre o aparecimento de determinadas doenças bucais com o surgimento e/ou agravamento da condição sistêmica (Constantin et al., 2018; Diawara et al., 2018; Vázquez-Sánchez et al., 2018).

Outro grande problema são as infecções relacionadas a faringe devido principalmente as faringotonsilites, que constituem uma das principais queixas no pronto-atendimento (Costa et al., 2018). A dor de garganta aguda é um dos problemas mais recorrentes no atendimento da clínica médica e é uma das principais causas de prescrição inadequada de antibióticos (van der Velden et al., 2020). Além disso, de acordo com a Organização Mundial de Saúde, a saúde bucal é um sinalizador chave da saúde geral e da qualidade de vida. Estima-se que aproximadamente, 3,58 bilhões de pessoas foram afetadas por doenças bucais no ano de 2016 e que 20\% das pessoas possuem lesões dentárias traumáticas (World Health Organization, 2020). Sendo assim, este trabalho teve como objetivo fazer uma revisão da literatura sobre o uso da planta Lippia sidoides Cham no tratamento de infecções relacionadas a faringe, cavidade oral e problemas odontológicos.

\section{Metodologia}

Trata-se de uma revisão de literatura com base nas seguintes etapas: identificação do tema e desenvolvimento da questão norteadora; estabelecimento de critérios de inclusão e exclusão, análise e seleção de estudos; interpretação de dados e resultados; apresentação da revisão. A pergunta norteadora foi: "Lippia sidoides Cham pode ser usada no tratamento de infecções relacionadas a faringe, cavidade oral e problemas odontológicos?" A pesquisa literária ocorreu a partir de artigos indexados em bibliotecas virtuais internacionais, Scientific Eletronic Library Online (SciELO), U.S. National Library of Medicine (PubMed), Google Acadêmico e Portal Periódicos Capes. Os descritores utilizados foram: Lippia sidoides Cham, infecções de faringe, infecções cavidade oral e odontologia, e seus correspondentes em inglês. Os critérios de inclusão foram: artigos originais publicados entre os anos de 2010 e 2020, em inglês ou português disponibilizados na integra. Os critérios de 
exclusão foram: trabalhos publicados em outro formato que não seja artigo, como teses e dissertações, artigos incompletos, artigos publicados em outro idioma que não os citados anteriormente.

\section{Resultados e Discussão}

\subsection{Fitoterapia}

Ao longo da evolução, o homem foi aprendendo a distinguir plantas para a sua alimentação e para o alívio de doenças, com isso muitos povos passaram a dominar o conhecimento do uso de plantas e ervas (Ferreira \& Pinto, 2010).

A fitoterapia é definida como a utilização de plantas medicinais para finalidades terapêuticas. Na saúde pública a fitoterapia é considerada um tipo de medicina alternativa. A principal característica da fitoterapia é o uso dessas plantas em inúmeras formas farmacêuticas, com o uso exclusivo de matérias-primas vegetais ativas (Avello \& Cisternas, 2010). Estima-se que $75 \%$ da população dos países emergentes, utilizam produtos naturais, enquanto nos países em desenvolvimento, aproximadamente 50\% da população faz o uso desses produtos (Carvalho et al., 2017).

Para a maioria da população a utilização das plantas medicinais é observada como uma prática integrativa e histórica. O consumo de produtos de origem natural apresentou um aumento relevante nos últimos anos, tanto nos países desenvolvidos quanto nos países em desenvolvimento (Santos et al., 2011; Sen \& Chakraborty, 2016; Yudice et al., 2018).

O entendimento sobre a capacidade curativa de algumas plantas é uma das formas de relação entre populações humanas e plantas. Além disso, as práticas relacionadas ao uso tradicional de plantas medicinais, são o que muitas comunidades têm como alternativa para o cuidado da saúde ou o tratamento de doenças (Fischer, Stumpf, \& Mariot, 2019; Giraldi \& Hanazaki, 2010). Nos últimos anos, as plantas cada vez mais têm sido o principal foco para estudos científicos, uma vez que possui uma grande diversidade de estruturas, propriedades químicas e substâncias naturalmente ativas (Pereira et al., 2013; Shakya, 2016).

A ciência moderna reconheceu a ação das plantas medicinais e, com isso incluiu na farmacoterapia moderna, uma série de medicamentos de origem vegetal, já conhecidos pelas sociedades antigas e utilizados ao longo dos milênios. Além disso, os avanços tecnológicos e as tendências de pesquisas, demonstram que os produtos naturais no futuro também estarão entre as fontes mais significativas de novos medicamentos (Atanasov et al., 2015; Petrovska, 2012).

Plantas medicinais aplicadas na saúde engloba a prevenção de doenças, a manutenção e recuperação da saúde, bem como a melhoria da qualidade de vida tanto de usuários quanto das comunidades (Alcantara et al., 2015). Dentre as razões relacionadas ao uso das plantas medicinais na atenção básica de saúde, estão o elevado custo dos medicamentos industrializados, a falta de acesso da população às assistências médica e farmacêutica e a tendência atual dos consumidores em usar produtos de origem natural (Gonçalves et al., 2013; WHO Report, 2019)O consumo de medicamentos provenientes de plantas tem crescido em todo o mundo nos últimos anos, principalmente no Brasil devido a sua grande biodiversidade, que é uma fonte potencial de novos medicamentos (Mazzari \& Prieto, 2014). A história do emprego dos fitoterápicos e plantas medicinais no campo dos serviços de atenção primária à saúde no Brasil, foi promovida por movimentos populares, diretrizes de várias conferências nacionais de saúde, e por orientações da OMS (Antonio et al., 2014). A utilização da fitoterapia na atenção básica à saúde possivelmente, representa além de uma diminuição de custos, o respeito pelos valores culturais. A OMS também admite a importância das plantas medicinais no tratamento e prevenção de inúmeras doenças, além de entender a importância a nível econômico (Avello \& Cisternas, 2010; Rosa et al., 2011).

No Brasil, a Política Nacional de Plantas Medicinais e Fitoterápicos, representa uma parte importante das Políticas Públicas de Saúde, meio ambiente, desenvolvimento econômico e social, funcionando como um dos elementos fundamentais de transversalidade na implementação de ações que possibilitam melhorias na qualidade de vida da população (Saúde, 2018). 
Adicionalmente, a Política Nacional de Plantas Medicinais e Fitoterápicos, por meio do decreto $\mathrm{n}^{\circ} 5.813$, de 22 de junho de 2006 estabelece diretrizes a fim de garantir o acesso seguro e o uso racional das plantas medicinas e fitoterápicos no Brasil, além de promover o uso sustentável (Brasil, 2016).

\subsection{Constituintes e propriedades químicas da Lippia sidoides Cham}

Os metabólitos secundários podem ser chamados de princípios ativos vegetais, formados a partir de produtos da fotossíntese. Em geral, essas substâncias servem como forma de defesa de muitas plantas e são responsáveis pelo seu efeito medicinal. Desta forma, essas ervas apresentam um grande potencial na área farmacêutica (Cardoso et al., 2019; Kochhar \& Gujral, 2020).

Essa planta possui outros componentes como, $\alpha$-tujeno, sabineno $\beta$-pineno, mirceno, $\alpha$-felandreno, $\alpha$-terpineno, $\rho$ cimeno, limoneno, 1,8-cineol $\gamma$-terpineno, terpin-4-ol, $\alpha$-terpineol, verbenona, metil-éter-timol, Neral, Timol, carvacrol, timol acetato, $\beta$-elemeno, (E)-cariofileno, $\alpha$-humuleno, dehidro-aromadendrano $\gamma$-muuroleno, amorfa-4,7-(11) -dieno, 7 -epi- $\alpha$ selineno e óxido de cariofileno ( Lima et al., 2011).

A Lippia sidoides possui diversos metabólitos secundários, sendo os majoritários o 5-isopropil-2-metil-fenol (carvacrol), p-cimeno e timol (Guimarães et al., 2014; Helenicy N.H. Veras et al., 2017). O carvacrol possui ação antiinflamatória, antioxidante, antifúngica e antibacteriana. Essa capacidade está relacionada com a interação que esses compostos têm com as membranas celulares de diferentes microrganismos. Devido a sua natureza hidrofóbica, esse monoterpeno interage com a bicamada lipídica da membrana citoplasmática e se alinha entre as cadeias de ácidos graxos, fazendo com que a estrutura da membrana se expanda e desestabilize, com isso aumenta a fluidez e permeabilidade para íons e prótons, fazendo com que ocorra uma perda do gradiente de íons, o que leva à morte celular bacteriana (Ciandrini et al., 2014; Marchese et al., 2018).

O timol é um fenol monoterpeno cristalino incolor e isômero do carvacrol. Constitui uma substância importante, devido as suas diversas atividades farmacológicas apresentadas na literatura, como cicatrizante, anti-inflamatório, antibacteriana e antifúngica. As plantas que possuem timol como um dos seus fitoconstituintes têm sido utilizadas na medicina tradicional para o tratamento de diversas patologias, como diabetes e doenças cardiovasculares (Marchese et al., 2016; Nagoor Meeran et al., 2017; Namdari et al., 2019).

Já foi comprovado que o timol e carvacrol possuem efeito bacteriostática sobre as cepas de Escherichia coli, dessa forma possuem o potencial de se tornarem antimicrobianos (D. S. de Lima et al., 2017). Alguns estudos já avaliaram a atividades antifúngica do timol. Silva e colaboradores (2017) comprovaram que o timol, na concentração de $5 \mu \mathrm{g} / \mathrm{mL}$, apresentou atividade frente a cepas de Candida spp (Silva; et al., 2017) isoladas da cavidade oral. Jia e colaboradores (2019) realizaram um estudo para avaliar a atividade antifúngica do timol e o carvacrol frente ao patógeno Botrytis cinerea. Os resultados indicaram que esses fitoconstituintes exibiram forte atividade contra o patógeno (Jia et al., 2019).

O p-cimeno também conhecido como 4-isopropiltolueno, é um monoterpeno encontrado nas plantas medicinais. Possui diversas atividades biológicas, como antioxidante, anti-inflamatório e antimicrobiano. Apesar da literatura descrever o p-cimeno como antimicrobiano, sua atividade é menos eficaz que outros fitoconstituintes, como o carvacrol, porém ele pode aumentar a atividade de outros antimicrobianos através de interações que provocam efeitos sinérgicos e aditivos (Marchese et al., 2017).

Em um estudo realizado por Yang e colaboradores (2014) foi proposta a interação do p-cimeno com compostos vegetais com propriedades antimicrobianas, linalol, terpinen-4-ol, terpinenol. O p-cimeno em combinação com essas outras moléculas apresentou efeitos aditivos contra Salmonella enterica, Staphylococcus aureus, Streptococcus sanguinis e S. mutans (Yang et al., 2014). 
Rattanachaikunsopon e Phumkhachorn (2010) realizaram um estudo para avaliar a atividade antimicrobiana do carvacrol e p-cimeno frente ao Vibrio cholerae. Nesse estudo, a bactéria foi inoculada em um suco de cenoura, posteriormente os fitoconstituintes foram adicionados ao suco isoladamente e em combinação. Os resultados mostraram que o carvacrol quando usado separadamente, exibiu um efeito inibitório frente a bactéria, mas o p-cimeno sozinho não teve atividade antimicrobiana. Porém, quando o p-cimeno e o carvacrol estavam juntos, foi percebido que o carvacrol teve seu efeito inibitório potencializado pelo p-cimeno. Assim, o carvacrol e o p-cimeno apresentam potencial como conservante contra $V$. cholerae em alimentos (Rattanachaikunsopon \& Phumkhachorn, 2010).

Aznar e colaboradores (2015) realizaram um estudo com o objetivo de observar a atividade antimicrobiana de nisina, carvacrol e p-cimeno frente a Candida lusitaniae. Os resultados demonstraram timol, carvacrol e p-cimeno inibiram completamente o crescimento da Candida lusitaniae (Aznar et al., 2015).

\subsection{Uso da Lippia sidoides Cham na medicina tradicional}

As folhas e flores da Lippia sidoides Cham constituem as principais partes da planta que são usadas na medicina popular administradas por meio de via oral ou através de emplastos. As principais formas farmacêuticas encontradas são decocto, infusão, tintura, colutório à base de óleo essencial, sabonete, gel e gel-creme ambos contendo óleo essencial ( Gomes et al., 2011).

Na medicina popular Lippia sidoides Cham é usada principalmente como antisséptico, além de ser utilizada no tratamento de infecções vaginais, e é aplicado topicamente na pele, mucosa, boca e garganta. É também usada como escabecida, antimicótico e para o tratamento de infecções da pele e couro cabeludo. O extrato aquoso tem sido principalmente usado como anti-inflamatório e cicatrizante (Brasil, 2021; De Farias et al., 2012; de Morais et al., 2016).

Além disso, também é usada na forma de sabão líquido e na forma de tintura hidroalcoólica, para o tratamento de feridas na pele e micoses. As folhas podem ser utilizadas sob a forma de chá abafado além de ser usada para o dor de cabeça e para torção (Coutinho et al., 2019; Leal et al., 2003; Monteiro et al., 2007).

As folhas também são usadas para o tratamento de acne, ferimentos, infecções na pele e do coro cabeludo. Além disso, também podem ser usadas na forma de infusão para o tratamento das infecções da boca e da garganta (Camelo et al., 2014). O extrato fluído das folhas é usado como solução de lavagem para lesões superficiais externas. Quando administrados por via tópica esse extrato possui efeito desinfetante e antimicrobiano. Além disso, a infusão de folhas e flores são usadas no tratamento de dor de estômago, cólica de bebê, indigestão, diarreia, azia, náusea, corrimentos vaginais e dores de origem menstrual (Oliveira et al., 2007; Saúde, 2018).

A literatura também descreve o uso do gel de alecrim pimenta na descontaminação das escovas dentais. Outra forma de uso popular da Lippia sidoides Cham, é na forma de sabão líquido que é utilizado para prevenir e tratar infecções causadas por fungos. Além disso, é comumente usada na forma de infusões e inalações para o tratamento de rinite alérgica e no tratamento de infecções vaginais, da boca e da garganta (de Medeiros et al., 2011; Oliveira et al., 2007).

\subsection{Uso da Lippia sidoides Cham no tratamento de infecções de faringe}

A faringite aguda é uma doença bastante frequente e as infecções virais são as causas mais comuns, porém as bactérias também são capazes de provocar a faringite. A principal bactéria relacionada a faringite é Streptococcus pyogenes, sendo responsável por $15 \%$ a $30 \%$ dos casos de faringite em crianças, e $5 \%$ a $20 \%$ em adultos. Além disso, os fungos também são capazes de induzir faringite em pacientes imunocomprometidos (Ferretti et al., 2016; Yeh, Li, \& Hsu, 2014).

A dor de garganta é uma condição comum relacionada com a infecção no trato respiratório superior e a recorrência dessa infecção pode resultar em amidalite crônica. A amidalite é causada principalmente por infecções bacteriana (Bathala \& 
Eccles, 2013; Yousef \& Yousef, 2010). Além disso, faringite e amidalite também podem ser causadas por outras bactérias, como Klebsiella pneumoniae, S. aureus, Pseudomonas aeruginosa, Neisseria gonorrhoeae, Mycoplasma pneumoniae, Fusobacterium spp., Chlamydia pneumoniae e Corynebacterium diphtheriae (Bathala \& Eccles, 2013; Brook, 2005; Gowrishankar et al., 2013; Matthews et al., 2020; Yousef \& Yousef, 2010).

Veras e colaboradores (2014) realizaram um estudo para avaliar a atividade antimicrobiana do óleo essencial da planta Lippia sidoides e de um dos seus constituintes majoritário, o timol, frente a oito cepas bacterianas, dentre elas, S. aureus, K. pneumoniae e $P$. aeruginosa. Observando os resultados foi possível perceber que tanto o óleo essencial da planta quanto o timol apresentaram atividade antibacteriana (CIM de $12 \mu \mathrm{g} / \mathrm{mL}$ para S. aureus, $256 \mu \mathrm{g} / \mathrm{mL}$ para K. pneumoniae e $512 \mu \mathrm{g} / \mathrm{mL}$ para P. aeruginosa) ( Veras et al., 2014).

Outro estudo realizado por Nader e colaboradores (2010) também evidenciou a ação dos extratos de diversas plantas, entre elas a Lippia sidoides, frente a cepas de S. aureus isoladas de diferentes fontes de uma propriedade leiteira, entre eles uma cepa isolada da garganta de um ordenhador (Nader et al., 2010). Castro e colaboradores (2011) analisaram a atividade antimicrobiana do óleo essencial de alecrim pimenta frente a isolados bacterianos, como por exemplo, S. aureus. Através dos resultados foi possível verificar que a CIM do óleo foi de $13 \mu \mathrm{L} / \mathrm{mL}$ frente a essa cepa testada. Já no resultado da concentração bactericida mínima (CBM), foi observado um efeito bactericida do óleo essencial dessa planta na concentração $25 \mu \mathrm{L} / \mathrm{mL}$ (Castro et al., 2011).

Vásquez-Sánchez e colaboradores (2018) avaliaram o óleo essencial de onze plantas frente a duas cepas de $S$. aureus (S8 e S10). O óleo essencial da Lippia sidoides foi o mais eficaz. Além disso, foi testado também a atividade antibiofilme e os resultados demonstraram que o óleo essencial da planta foi o único antimicrobiano capaz de erradicar completamente os biofilmes da cepa S10 (Vázquez-Sánchez et al., 2018). A Figura 1 apresenta algumas formas de utilização do extrato da Lippia sidoides e suas indicações terapêuticas.

Figura 1 - Formas de aplicação e indicações terapêuticas do extrato de Lippia sidoides Cham.

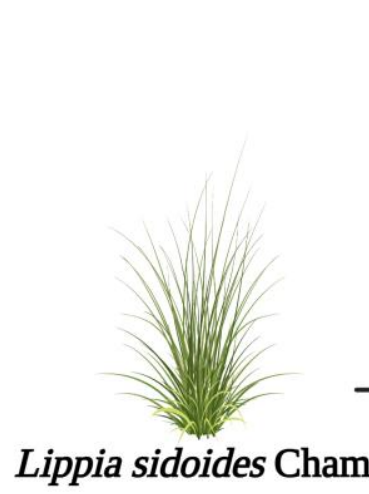

Lippia sidoides Cham

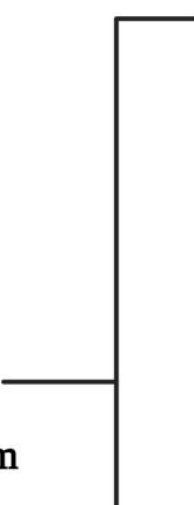

-

Infusão

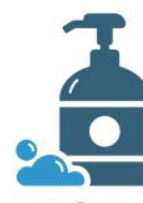

Sabão

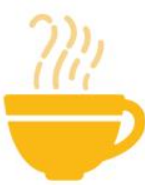

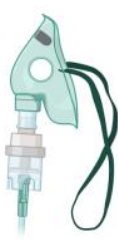

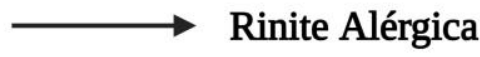

Inalação

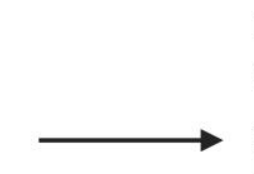

Dor de estômago

Cólica

Azia

Diarreia

Corrimento vaginal

Infecções

Fonte: Autoria própria. 


\subsection{Uso da Lippia sidoides Cham no tratamento infecções de cavidade oral}

A cavidade oral, também chamada de boca, é formada pelas bochechas, palato duro, palato mole e língua. Essa cavidade possui diversos habitats diferentes e o desequilíbrio da flora microbiana contribui para o surgimento de doenças bucais (Gao et al., 2018; Tortora \& Derrickson, 2016). Essa cavidade é colonizada por microrganismos que causam doenças, como bactérias, fungos e protozoários. Atualmente, os microrganismos da cavidade oral desenvolveram resistência a antibióticos e produtos químicos sintéticos. Dessa forma, muitos desses produtos tem a sua ação reduzida, com isso, aumentou à atenção para as plantas medicinais para serem usadas como antimicrobianos alternativos e úteis e serem agregados aos enxaguantes bucais e cremes dentais (Chinsembu, 2016).

A candidíase oral é uma infecção fúngica comum da boca, causada por diferentes espécies de Candida, onde a principal espécie responsável por essa afecção é a Candida albicans. As espécies Candida krusei e Candida dubliniensis também são encontradas na cavidade oral (Anibal et al., 2010; Faria \& Iecher, 2014; Singh et al., 2014).

Além das candidíases orais, outras infecções fúngicas oportunistas na mucosa oral são ocasionadas por Aspergillus fumigatus e Cryptococcus neoformans. Em pacientes imunocomprometidos, Sacharomyces cerevisiae também pode causar infecção fúngica na cavidade oral (Alves et al., 2016; Deepa et al., .

Batista e colaboradores (2013) testaram o extrato hidroalcóolico e o óleo essencial da Lippia sidoides frente a cepas de Candida albicans, Candida parapsilosis, Candida tropicalis e Candida guillermondii. Nesse estudo, tanto o extrato hidroalcóolico quanto o óleo essencial apresentaram atividade inibitória frente a todas as espécies de Candida testadas (Batista et al., 2013). A Figura 2 mostra esquema da eficácia do extrato da Lippia sidoides em cepa de Candida sp.

Figura 2 - Esquema dos testes de avaliação da atividade antifúngica do extrato de Lippia sidoides Cham frente a Candida sp.

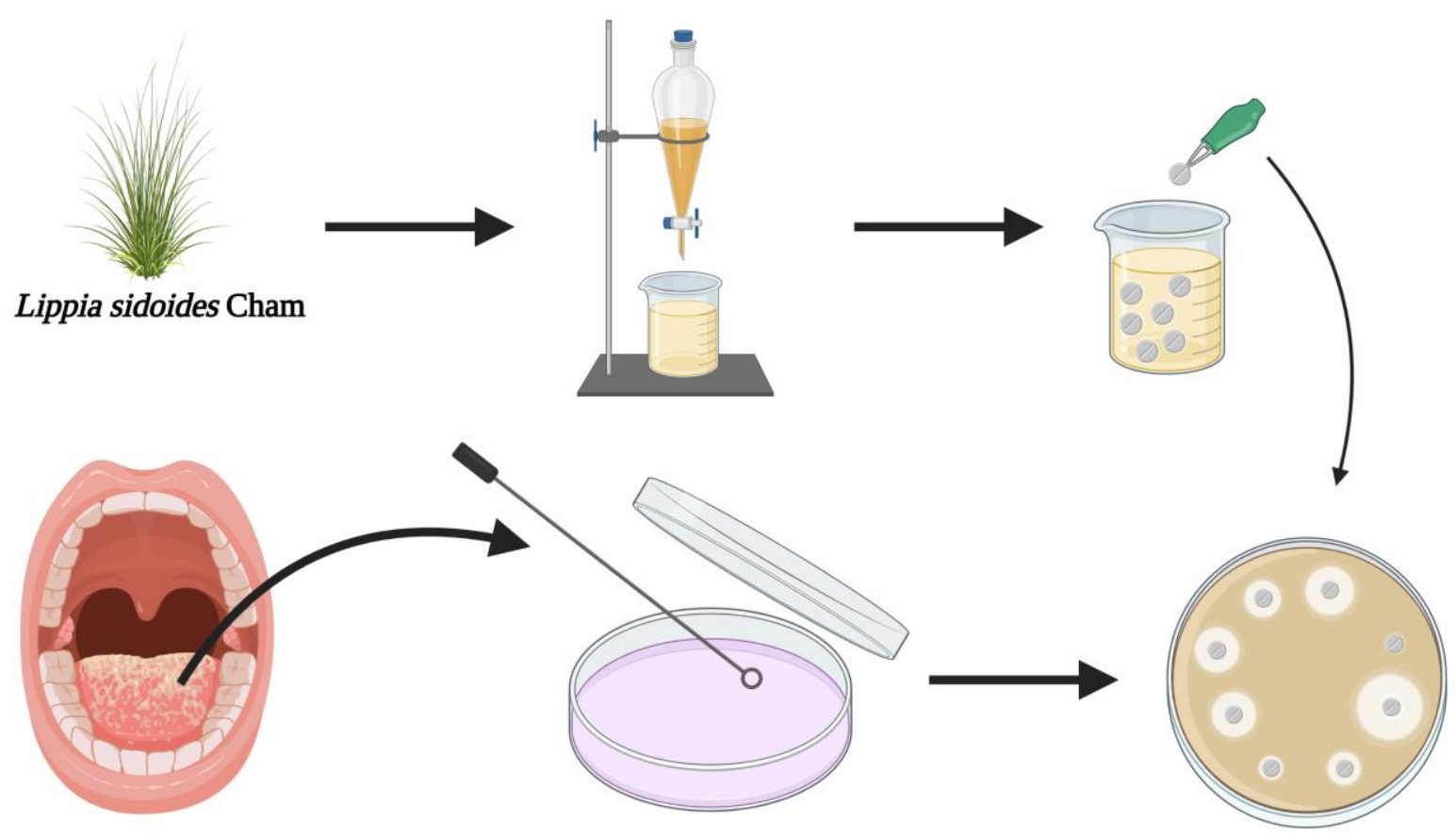

Candidíase

Fonte: Autoria própria.

Ahmad et al. (2013) analisaram a atividade dos componentes majoritários da planta alecrim-pimenta, o timol e carvacrol, frente a cepas de Candida e avaliaram a combinação desses compostos com o fluconazol. Ambos os monoterpenos 
apresentaram ação inibitória frente a todas as cepas estudadas. Os resultados em relação a combinação dos compostos com o antibiótico mostraram que as combinações timol-fluconazol e carvacrol-fluconazol foram sinérgicas frente a quase todos os isolados (Ahmad et al., 2013).

Funari e colaboradores (2012) avaliaram a atividade antifúngica de compostos isolados dos extratos de espécies do gênero Lippia spp. frente a cepas de Candida albicans, Candida krusei, Candida parapsilosis e Cryptococcus neorformans. Os compostos detectados no extrato dessa planta foram Forsitosídeo B, Verbascosídeo, Asebogenina. Quando analisado os resultados da ação desses extratos, foi possível concluir que esses compostos inibiram o crescimento de todas as cepas, porém foram mais ativos frente a cepa de C. neoformans (Funari et al., 2012).

Brito e colaboradores (2015) realizaram um estudo para analisar a atividade antifúngica do óleo essencial do alecrimpimenta e do timol frente a cepas de Candida albicans, Candida krusei e Candida tropicalis. Os resultados dessa pesquisa revelaram o potencial antifúngico e o efeito inibidor dessa planta e do seu composto, uma vez que, a CIM do óleo essencial da planta frente às cepas variou entre 64 e $256 \mu \mathrm{g} / \mathrm{mL}$, enquanto a CIM do timol variou entre 32 e $64 \mu \mathrm{g} / \mathrm{mL}$. Além disso a concentração fungicida mínima (CFM) do óleo essencial foi determinada entre 128 e $512 \mu \mathrm{g} / \mathrm{mL}$ e para o timol foram encontrados valores entre 64 e $128 \mu \mathrm{g} / \mathrm{mL}$ (Brito et al., 2015).

O estudo realizado por Morais e colaboradores (2016) testou o óleo essencial e frações hexânica (HF), diclorometano (DF), acetato de etila (EAF) e aquosa (AF) da Lippia sidoides frente a cepas de fungos, dentre elas cepas, C. parapsilosis, $C$. albicans, $C$. neorformans, e Cryptococcus gattii. O óleo essencial apresentou atividade inibitória frente a C. parapsilosis, $C$. gatti e C. neoformans, porém das frações, apenas a HF teve ação inibitória frente as cepas estudadas (de Morais et al., 2016).

\subsection{O uso de plantas medicinais na odontologia}

A utilização de produtos naturais na odontologia representa uma alternativa acessível e eficiente na prevenção e combate de diversas patologias da cavidade oral. Em 2008, por meio da resolução $n^{\circ}$ 082/2008-CFO, foi reconhecida e regulamentada pelo Conselho Federal de Odontologia o uso da fitoterapia pelo cirurgião dentista, como prática integrativa e complementar à saúde bucal (Evangelista et al., 2012).

Pesquisas com produtos naturais na área odontológica como controle de biofilme dental e outras infecções bucais têm crescido, objetivando buscar novos produtos com maior atividade terapêutica, menor toxicidade, além de apresentarem valor mais acessível à população (Anibal et al., 2010).

O efeito das plantas medicinais, como elemento tanto nos géis dentifrícios, como nos enxaguantes bucais, têm sido analisados para o tratamento de infecções bucais, como a gengivite. Os produtos de higiene bucal com ativos derivados de vegetal vêm sendo cada vez mais estudados com protocolos mais modernos, que demostrem a ação da utilização de determinadas plantas no tratamento das doenças bucais (Cordeiro et al., 2006; Gomes et al., 2020).

Utilização de fitoterápicos na odontologia demonstra ser uma vantagem, uma vez que possui custo acessível, fácil manipulação e menor efeito colateral quando comparados a medicamentos que não são fitoterápicos. Na área odontológica, as plantas medicinais têm sido usadas como agentes anti-inflamatório, analgésicos, sedativos e, como irrigantes endodônticos (Groppo et al., 2008; Oliveira et al., 2018).

Plantas com propriedades medicinais são consideradas uma fonte eficiente e útil no tratamento para vários processos de doenças. Extratos de plantas têm sido cada vez mais utilizados com êxito na odontologia contra algumas infecções bucais, como as placas microbianas, além de também auxiliarem no processo de cicatrização. As plantas medicinais, também têm sido estudadas para prevenção de problemas odontológicos, principalmente cárie e doença periodontal, que é um estágio avançado da gengivite (Lobo et al., 2015). A cárie é uma doença infecciosa de origem multifatorial e diversos estudos demostram que $S$. mutans está relacionada com a cárie dental em humanos. Além disso, bactérias do gênero Lactobacilus estão associadas com o 
avanço da cárie em humanos, assim com Streptococcus mitis, Streptococcus sanguinis e Streptococcus salivares, que também são consideradas bactérias cariogênicas (Losso et al., 2009; Machado et al., 2016; Vicente et al., 2010). A doença periodontal, também chamada de gengivite têm como agente etiológico o acúmulo do biofilme, além da debilidade no sistema imunológico do paciente e a morfologia do tecido gengival também podem contribuir com o surgimento dessa afecção bucal (Granvillegarcia et al., 2010). A figura 3 mostra as principais indicações do extrato de Lippia sidoides na cavidade oral.

Figura 3 - Usos terapêuticos do extrato de Lippia sidoides Cham na cavidade oral.

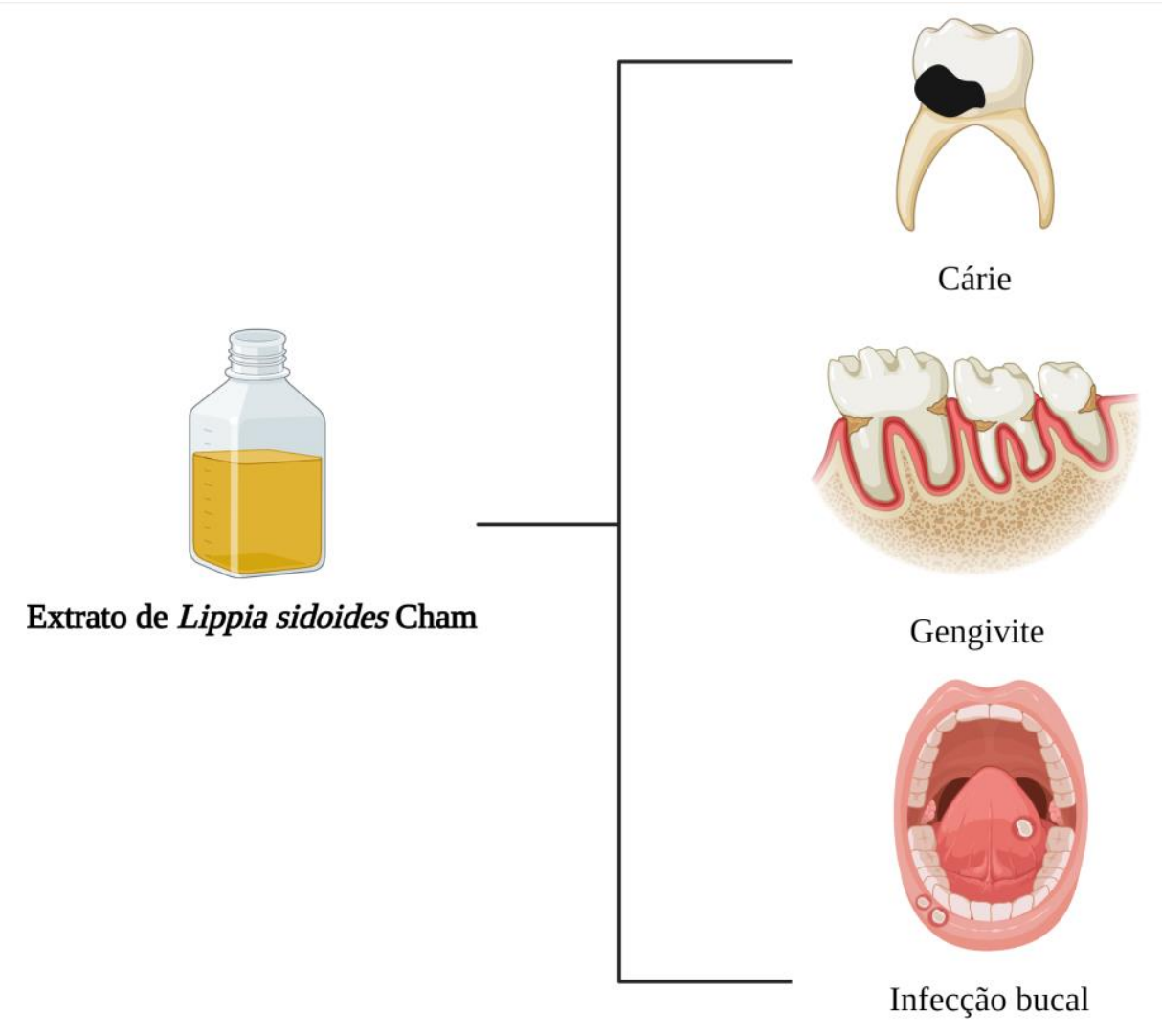

Fonte: Autoria própria.

Lobo e colaboradores (2011) realizaram uma pesquisa in vivo para avaliar a atividade de um gel contendo óleo essencial de Lippia sidoides em diferentes concentrações $(0,8 \%, 1 \%, 1,2 \%, 1,4 \%)$ e de um enxaguante bucal, também contendo óleo essencial da planta em diferentes concentrações $(0,6 \%, 0,8 \%, 1 \%, 1,2 \%)$, frente a S. mutans. Os resultados revelaram que a maior redução percentual de $S$. mutans, foi percebida na concentração de $0,8 \%$ do enxaguante bucal e na concentração de 1,4\% do gel. Dessa forma, as formulações mostraram ser eficientes contra S. mutans (Lobo et al., 2011).

Outro estudo de Lobo e colaboradores (2014) realizou um ensaio clínico randomizado para avaliar a ação de três formulações do óleo essencial de Lippia sidoides, pasta de dente a 1,4\%, gel a 1,4\% e colutório a $0,8 \%$, frente a Streptococcus mutans de crianças com cárie. Os resultados demonstraram que o creme dental reduziu os níveis de S. mutans após 5 dias de tratamento, e os níveis de $S$. mutans permaneceram baixos e não retornaram aos valores basais durante a análise subsequente. Porém o gel e o colutório não reduziram efetivamente os níveis de $S$. mutans em crianças com cárie dentária (Lobo et al., 2014).

Pereira e colaboradores (2013) realizaram um ensaio clínico para avaliar a eficácia de um gel contendo 10\% do óleo essencial de alecrim-pimenta, no controle de placa bacteriana e gengivite. Os resultados mostraram que o gel não apresentou 
nenhum efeito adverso. Além disso, o gel foi eficiente na redução de placas bacterianas, como também frente a gengivite, em 50\% e 40\%, respectivamente (Pereira et al., 2013).

Veras e colaboradores (2014) analisaram a atividade antimicrobiana do óleo essencial da Lippia sidoides e do timol sobre o biofilme de Enterococcus faecalis que foi isolada de canais radiculares dentários. O óleo essencial da planta e o timol reduziram o biofilme de E. faecalis com tempo de exposição de 30 e 60 min, nas concentrações de 2,5\% e 10\%. Além disso, não houve diferença estatística do efeito entre o óleo essencial e timol (Veras et al., 2014).

Rodrigues e colaboradores (2013) realizaram um estudo clínico com o objetivo de avaliar o efeito antiplaca de enxaguante bucal com 10\% do óleo essencial da Lippia sidoides. O enxaguante bucal contendo 10\% do óleo da planta teve efeito antiplaca semelhante ao controle positivo, digluconato de clorexidina $0,12 \%$. Além disso, quando testados em uma dentição sem placa bacteriana, o enxaguante com o óleo essencial apresentou significativamente menos acúmulo de placa em relação aos resultados do grupo controle (Rodrigues et al., 2013).

Albuquerque e colaboradores (2013) realizaram um estudo para avaliar o efeito antiaderente do extrato da Lippia sidoides Cham frente a microrganismos do biofilme dental, como, por exemplo, S. mutans, S. sanguinis e Lactobcillus casei. Os resultados mostraram que o extrato apresentou atividade antiaderente frente a todas as cepas testadas. Além disso, o efeito antiaderente do extrato da planta frente a $S$. mutans é semelhante ao efeito da clorexidina, antibiótico padrão utilizado para a cavidade oral (Albuquerque et al., 2012).

\section{Conclusão}

A Lippia sidoides Cham é uma das espécies de plantas medicinais usadas na medicina popular para o tratamento de diversas doenças, entre elas, dor de garganta e infecções na cavidade oral. O potencial farmacêutico dessa planta está relacionado, principalmente, aos seus constituintes químicos majoritários, o timol e o carvacrol, que possuem propriedades terapêuticas descritas em diversos trabalhos, como ação anti-inflamatória, antioxidante, antifúngica e antibacteriana.

Diversos estudos farmacológicos comprovam a atividade antibacteriana e antifúngica dos extratos e do óleo essencial dessa planta frente a cepas de bactérias cariogênicas, como S. mutans e frente a cepas de diferentes espécies de Candida, que é o principal fungo relacionado as infecções da cavidade oral. Além disso, os estudos confirmam a eficácia de produtos odontológicos contendo óleo essencial da Lippia sidoides frente as principais infecções odontológicas.

Assim, Lippia sidoides Cham é uma espécie vegetal bastante utilizada na medicina tradicional e comercializada para o tratamento de infecções relacionadas a faringe, cavidade oral e problemas odontológicos. Estudos futuros com avaliação da sua eficácia em outras áreas são necessários e podem apresentar uma nova alternativa de tratamento de inúmeras patologias.

\section{Agradecimentos}

F.A.S.A. agradece à Fundação de Apoio à Ciência e Tecnologia do Estado de Pernambuco -FACEPE pela bolsa de iniciação científica. R.G.A.R.R. agradece ao Conselho Nacional de Desenvolvimento Científico e Tecnológico -CNPq. I.M.F.C. agradece ao CPNq (426065/2018-2) por parte do apoio financeiro deste trabalho.

\section{Referências}

Ahmad, A., Khan, A., \& Manzoor, N. (2013). Reversal of efflux mediated antifungal resistance underlies synergistic activity of two monoterpenes with fluconazole. European journal of pharmaceutical sciences: official journal of the European Federation for Pharmaceutical Sciences, 48(1-2), 80-86. Netherlands.

Albuquerque, A., Pereira, M. S. V, Silva, D. F., Pereira, L. F., Viana, F. A. C., Higino, J., \& Barbosa, M. (2012). The anti-adherence effect of Lippia sidoides Cham. Extract against microorganisms of dental biofilm. Revista Brasileira de Plantas Medicinais, 15, 41-46.

Alcantara, R. G. L., Joaquim, R. H. V. T., \& Sampaio, S. F. (2015). Plantas medicinais: O conhecimento e uso popular. Revista de APS, $18(4), 470-482$. 
Alves, L. C., Cano, J. F., Galafassi, D., Elsemann, E. B., Elsemann, R. B., \& Gazzoni, A. F. (2016). Infecção fúngica na cavidade oral ocasionada por Saccharomyces cerevisiae: Relato de caso.

Anibal, P. C., de Cássia Orlandi Sardi, J., Peixoto, I. T. A., de Carvalho Moraes, J. J., \& Höfling, J. F. (2010). Conventional and alternative antifungal therapies to oral candidiasis. Brazilian journal of microbiology: [publication of the Brazilian Society for Microbiology], 41(4), 824-831. Sociedade Brasileira de Microbiologia. https://pubmed.ncbi.nlm.nih.gov/24031562

Antonio, G. D., Tesser, C. D., \& Moretti-Pires, R. O. (2014). Phytotherapy in primary health care. Revista de Saúde Pública, 12(1), 58-68.

Atanasov, A. G., Waltenberger, B., Pferschy-Wenzig, E. M., Linder, T., Wawrosch, C., Uhrin, P., Temml, V., et al. (2015). Discovery and resupply of pharmacologically active plant-derived natural products: A review. Biotechnology Advances, 33(8), 1582-1614. The Authors. http://dx.doi.org/10.1016/j.biotechadv.2015.08.001

Avello, M. L., \& Cisternas, I. F. (2010). Fitoterapia, sus orígenes, características y situación en Chile. Revista Medica de Chile, 138(10), 1288-1293.

Aznar, A., Fernández, P. S., Periago, P. M., \& Palop, A. (2015). Antimicrobial activity of nisin, thymol, carvacrol and cymene against growth of Candida lusitaniae. Food science and technology international = Ciencia y tecnologia de los alimentos internacional, 21(1), 72-79. United States.

Bathala, S., \& Eccles, R. (2013). A review on the mechanism of sore throat in tonsillitis. The Journal of Laryngology \& Otology, 127(3), 227-232. Cambridge University Press. https://www.cambridge.org/core/article/review-on-the-mechanism-of-sore-throat-in-tonsillitis/5C62DCDC60E6FAB88E1C4D6A62A4681A

Batista, R., Silva, G., Machado, S., \& Vieira, K. (2013). Atividade antifúngica de alecrim-pimenta (Lippia sidoides Cham.) sobre Candida spp. Agropecuária Técnica, 34, 44-49.

Brasil. (2009). Ministério da Saúde RENISUS. Relação nacional de plantas medicinais de interesse ao SUS. Espécies vegetais. MS elabora Relação de Plantas Medicinais de Interesse ao SUS. Retrieved January 5, 2022, from http://portalarquivos2.saude.gov.br/images/pdf/2014/maio/07/renisus.pdf

Brasil. (2016). Política e Programa Nacional de Plantas Medicinais e Fitoterápicos. Secretaria de Ciência, Tecnologia e Insumos Estratégicos. Departamento de Assistência Farmacêutica. http://bvsms.saude.gov.br/bvs/publicacoes/politica_programa_nacional_plantas_medicinais_fitoterapicos.pdf

Brasil. (2021). Formulário de Fitoterápicos Agência Nacional de Vigilância Sanitária-Anvisa $2^{\mathrm{a}}$ EDIÇÃO. Agência Nacional de Vigilância Sanitária, 223. https://www.gov.br/anvisa/pt-br

Brito, D. I. V., Morais-Braga, M. F. B., Cunha, F. A. B., Albuquerque, R. S., Carneiro, J. N. P., Lima, M. S. F., Leite, N. F., et al. (2015). Análise fitoquímica e atividade antifúngica do óleo essencial de folhas de Lippia sidoides Cham. e do Timol contra cepas de Candida spp. Revista Brasileira de Plantas Medicinais, 17(4), 836-844.

Brook, I. (2005). The role of anaerobic bacteria in tonsillitis. International journal of pediatric otorhinolaryngology, 69, 9-19.

Camelo, A. L. M., Oliveira, F. C. de, Silva, F. F. M. da, Souza, F. T. C. de, \& Longhinotti, E. (2014). Análise Comparativa Da Composição Química Volátil De Amostras Do Alecrim in Natura Com a Disponível Comercialmente. Holos, 5, 191.

Cardoso, J. C., de Oliveira, M. E. B. S., \& Cardoso, F. de C. I. (2019). Advances and challenges on the in vitro production of secondary metabolites from medicinal plants. Horticultura Brasileira, 37(2), 124-132.

Carvalho, L. G., Costa, S. L. da, \& Costa, D. F. A. (2017). Principais fitoterápicos e demais medicamentos utilizados no tratamento de ansiedade e depressão Main phytotherapics and other drugs used to treat anxiety and depression Principales medicamentos a base de hierbas y otros fármacos utilizados para tratar la, (1), 2021. https://orcid.org/0000-0002-7317-2829

Castro, C. ., Ribeiro, J. ., Diniz, T. ., Almeida, A. ., Ferreira, L. ., Martins, E. ., \& Duarte, E. . (2011). Antimicrobial activity of Lippia sidoides Cham. (Verbenaceae) essential oil against Staphylococcus aureus and Escherichia coli. Revista Brasileira de Plantas Medicinais, 13(3), $293-297$.

Chinsembu, K. C. (2016). Plants and other natural products used in the management of oral infections and improvement of oral health. Acta tropica, 154, 618. Netherlands.

Ciandrini, E., Campana, R., Federici, S., Manti, A., Battistelli, M., Falcieri, E., Papa, S., et al. (2014). In vitro activity of Carvacrol against titanium-adherent oral biofilms and planktonic cultures. Clinical oral investigations, 18(8), 2001-2013. Germany.

Constantin, M., Forna, D. A., Budacu, C., Caraiane, A., Raftu, G., \& Forna, N. C. (2018). Oral tumors having the origins in multiple tissues, non-differentiated or differentiated. Revista de Chimie, 69(10), 2895-2898.

Cordeiro, C., Sacramento, L., Corrêa, M., Pizzolitto, A., \& Bauab, T. (2006). Análise farmacognóstica e atividade antibacteriana de extratos vegetais empregados em formulação para a higiene bucal. Revista Brasileira De Ciencias Farmaceuticas - RBCF, 42.

Costa, G. A., Alves, W. R., Almeida, G. A., \& Lima, M. M. (2018). Diagnóstico diferencial das faringotonsilites na infância. Revista Médica de Minas Gerais, 28(Supl.6), e-S280612.

Coutinho, A. L., Lucena, R. F. P. de, \& Bonifácio, K. M. (2019). Conhecimento e utilização de plantas mágico-religiosas por rezadeiras do semiárido da Paraíba, Nordeste do Brasil. Revista Ibero-Americana de Ciências Ambientais, 9(8), 235-248.

Deepa, A., Nair, B. J., Sivakumar, T., \& Joseph, A. P. (2014). Uncommon opportunistic fungal infections of oral cavity: A review. Journal of oral and maxillofacial pathology: JOMFP, 18(2), 235-243. Medknow Publications \& Media Pvt Ltd. https://pubmed.ncbi.nlm.nih.gov/25328305

Diawara, O., Ba, B., Ba, M., Niang, A., Kane, A. S. T., Kayentao, K., Nimaga, A., et al. (2018). Oral hygiene: Knowledge and practices of students in three quranic schools in Koutiala, Mali. Pesquisa Brasileira em Odontopediatria e Clinica Integrada, 18(1), 1-6. 
Evangelista, S., Sampaio, F., Parente, R., \& Fulgência, M. (2012). Phytotherapics in Odontology: ethnobotanical study in Manaus. Revista Brasileira de Plantas Medicinais, 15, 513-519.

Faria, M. B. B., \& Iecher, M. G. (2014). Produtos naturais como nova alternativa terapêutica para o tratamento de candidíase bucal. Revista UNINGÁ Review, 20(1), 103-107.

De Farias, E. M. F. G., Ximenes, R. M., Magalhães, L. P. M. H., Chiappeta, A. D. A., De Sena, K. X. D. F. R., \& De Albuquerque, J. F. C. (2012). Antifungal activity of Lippia sidoides Cham. (Verbenaceae) against clinical isolates of Candida species. Journal of Herbal Medicine, 2(3), 63-67.

Ferreira, V. F., \& Pinto, A. C. (2010). A fitoterapia no mundo atual. Quimica Nova, 33(9), 1829.

Ferretti, J. J., Stevens, D. L., \& Fischetti, V. A. (Eds.). (2016). No Title. Oklahoma City (OK).

Fischer, C. H., Stumpf, E. R. T., \& Mariot, M. P. (2019). A construção de uma prática pedagógica a partir do conhecimento familiar sobre plantas medicinais. Revista Educar Mais, 3(1), 56-68.

Funari, C. S., Gullo, F. P., Napolitano, A., Carneiro, R. L., Mendes-Giannini, M. J. S., Fusco-Almeida, A. M., Piacente, S., et al. (2012). Chemical and antifungal investigations of six Lippia species (Verbenaceae) from Brazil. Food chemistry, 135(3), 2086-2094. England.

Gao, L., Tiansong, X., Huang, G., Jiang, S., Gu, Y., \& Chen, F. (2018). Oral microbiomes: more and more importance in oral cavity and whole body. Protein \& cell, 9 .

Giraldi, M., \& Hanazaki, N. (2010). Uso e conhecimento tradicional de plantas medicinais no Sertão do Ribeirão, Florianópolis, SC, Brasil. Acta Botanica Brasilica, 24(2), 395-406.

Gomes, M. S., Pereira de Mendonça, A. K., Cordeiro, T. O., \& Barbosa, M. M. (2020). Uso De Plantas Medicinais Na Odontologia: Uma Revisão Integrativa. Revista de Ciências da Saúde Nova Esperança, 18(2), 118-126.

Gomes, S. F. L., de Lima Nogueira, P. C., \& de Souza Moraes, V. R. (2011). Aspectos químicos e biológicos do gênero Lippia enfatizando Lippia gracilis Schauer. Eclética Química, 36, 64-77.

Gonçalves, M. D. M. M., Cajaiba, R. L., Santos, W. B. dos, Sousa, E. S. de, Martins, J. D. S. C., Pereira, K. S., \& Sousa, V. D. A. (2018). Estudo etnobotânico do conhecimento e uso de plantas medicinais em Santa Luzia, Maranhão, Brasil. Revista Ibero-Americana de Ciências Ambientais, 9(5), 12-21.

Gonçalves, N. D. C. T., Vila, M. G. M., \& Chave, D. S. de A. (2013). Políticas de Saúde para a Fitoterapia no Brasil. Revista Cubana de Plantas Medicinales, $18(6)$.

Gowrishankar, S., Thenmozhi, R., Balaji, K., \& Pandian, S. K. (2013). Emergence of methicillin-resistant, vancomycin-intermediate Staphylococcus aureus among patients associated with group A Streptococcal pharyngitis infection in southern India. Infection, genetics and evolution: journal of molecular epidemiology and evolutionary genetics in infectious diseases, 14, 383-389. Netherlands.

Granville-garcia, A. F., Maria, A., Barbosa, F., \& Siqueira, M. J. (2010). Caries , gingivitis and oral hygiene in preschool children, 469-473.

Groppo, F. C., Bergamaschi, C. de C., Cogo, K., Franz-Montan, M., Motta, R. H. L., \& de Andrade, E. D. (2008). Use of phytotherapy in dentistry. Phytotherapy research: PTR, 22(8), 993-998. England.

Guimarães, L. G. de L., Cardoso, M. das G., Souza, R. M. de, Zacaroni, A. B., \& Santos, G. R. dos. (2014). Óleo essencial de Lippia sidoides nativas de Minas Gerais: composição, estruturas secretoras e atividade antibacteriana. Revista Ciência Agronômica, 45(2), 267-275.

Jia, C., Zhang, J., Yu, L., Wang, C., Yang, Y., Rong, X., Xu, K., et al. (2019). Antifungal Activity of Coumarin Against Candida albicans Is Related to Apoptosis. Frontiers in Cellular and Infection Microbiology, 8, 445. https://www.frontiersin.org/article/10.3389/fcimb.2018.00445

Kochhar, S. L., \& Gujral, S. K. (2020). Secondary Plant Metabolites. Plant Physiology, 590-610.

Leal, L. K. A. M., Oliveira, V. M., Araruna, S. M., Miranda, M. C. C., \& Oliveira, F. M. A. (2003). Análise de timol por CLAE na tintura de Lippia sidoides Cham. (alecrim-pimenta) produzida em diferentes estágios de desenvolvimento da planta. Revista Brasileira de Farmacognosia, 13, 9-11.

Lima, D. S. de, Lima, J. C., Calvacanti, R. M. C. B., Santos, B. H. C. dos, \& Lima, I. O. (2017). Estudo da atividade antibacteriana dos monoterpenos timol e carvacrol contra cepas de Escherichia coli produtoras de $\beta$-lactamases de amplo espectro. Revista Pan-Amazônica de Saúde, 8(1), 17-21.

Lima, L. O., \& Gomes, E. C. (2014). Alimento ou medicamento? Espécies vegetais frente à legislação Brasileira. Revista Brasileira de Plantas Medicinais, 16(3), 771-782.

Lima, R. K., Cardoso, M. das G., Moraes, J. C., Carvalho, S. M., Rodrigues, V. G., \& Guimarães, L. G. L. (2011). Chemical composition and fumigant effect of essentialoil of Lippia sidoides Cham. and monoterpenes against Tenebrio molitor (L.) (coleoptera: tenebrionidae). Ciência e Agrotecnologia, 35(4), 664671.

Lobo, P. L. D., Fonteles, C. S. R., de Carvalho, C. B. M., do Nascimento, D. F., da Cruz Fonseca, S. G., Jamacaru, F. V. F., \& de Moraes, M. E. A. (2011). Dose-response evaluation of a novel essential oil against Mutans streptococci in vivo. Phytomedicine: international journal of phytotherapy and phytopharmacology, 18(7), 551-556. Germany.

Lobo, P. L. D., Fonteles, C. S. R., Marques, L. A. R. V., Jamacaru, F. V. F., Fonseca, S. G. da C., de Carvalho, C. B. M., \& de Moraes, M. E. A. (2014). The efficacy of three formulations of Lippia sidoides Cham. essential oil in the reduction of salivary Streptococcus mutans in children with caries: a randomized, double-blind, controlled study. Phytomedicine : international journal of phytotherapy and phytopharmacology, 21(8-9), 1043-1047. Germany.

Lobo, P. L. D., Marques, L. A. R. V., Gurgel, M. F., Soares, J. L., Fonseca, S. G. da C., \& Moraes, M. E. A. de. (2015). Atividade Farmacológica do Óleo Essencial de Lippia sidoides em Odontologia: Uma Revisão de Literatura. Saúde e Pesquisa, 8(2), 373. 
Losso, E. M., Tavares, M. C. R., Da Silva, J. Y. B., \& Urban, C. D. A. (2009). Severe early childhood caries: An integral approach. Jornal de Pediatria, 85(4), 295-300.

Machado, B., Pulcino, T., Silva, A., Melo, D., Silva, R., \& Mendonca, I. (2016). Propolis as an alternative in prevention and control of dental cavity. Journal of Apitherapy, 1(2), 47.

Madaleno, I. M. (2011). Plantas da medicina popular de São Luís, Brasil. Boletim do Museu Paraense Emílio Goeldi. Ciências Humanas, 6(2), $273-286$.

Marchese, A., Arciola, C. R., Barbieri, R., Silva, A. S., Nabavi, S. F., Tsetegho Sokeng, A. J., Izadi, M., et al. (2017). Update on Monoterpenes as Antimicrobial Agents: A Particular Focus on p-Cymene. Materials (Basel, Switzerland), 10(8).

Marchese, A., Arciola, C. R., Coppo, E., Barbieri, R., Barreca, D., Chebaibi, S., Sobarzo-Sánchez, E., et al. (2018). The natural plant compound carvacrol as an antimicrobial and anti-biofilm agent: mechanisms, synergies and bio-inspired anti-infective materials. Biofouling, 34(6), 630-656. Taylor \& Francis. https://doi.org/10.1080/08927014.2018.1480756

Marchese, A., Orhan, I. E., Daglia, M., Barbieri, R., Di Lorenzo, A., Nabavi, S. F., Gortzi, O., et al. (2016). Antibacterial and antifungal activities of thymol: A brief review of the literature. Food chemistry, 210, 402-414. England.

Martins, S. M., Silva, Z. D. da, Ferreira, A. F. A., Diniz, M. V. L., Cahu, A. K. M., Lima, R. S., \& Moura, L. M. de. (2020). Fitoterapia: uma opção para o tratamento odontológico. Anais do I Congresso da Liga Acadêmica de Introdução a Odontologia. www.even3.com.br/Anais/ICLAIO2020/292150FITOTERAPIA--UMA-OPCAO-PARA-O-TRATAMENTO-ODONTOLOGICO

Matthews, D., Adegoke, O., \& Shephard, A. (2020). Bactericidal activity of hexylresorcinol lozenges against oropharyngeal organisms associated with acute sore throat. BMC research notes, 13(1), 99.

Mazzari, A. L. D. A., \& Prieto, J. M. (2014). Herbal medicines in Brazil: Pharmacokinetic profile and potential herb-drug interactions. Frontiers in Pharmacology, 5 JUL(July), 1-12.

de Medeiros, M. das G. F., da Silva, A. C., Citó, A. M. das G. L., Borges, A. R., de Lima, S. G., Lopes, J. A. D., \& Figueiredo, R. C. B. Q. (2011). In vitro antileishmanial activity and cytotoxicity of essential oil from Lippia sidoides Cham. Parasitology international, 60(3), 237-241. Netherlands.

Monteiro, M. V. B., de Melo Leite, A. K. R., Bertini, L. M., de Morais, S. M., \& Nunes-Pinheiro, D. C. S. (2007). Topical anti-inflammatory, gastroprotective and antioxidant effects of the essential oil of Lippia sidoides Cham. leaves. Journal of ethnopharmacology, 111(2), 378-382. Ireland.

de Morais, S., Oliveira, T., de Oliveira, L., Tresvenzol, L., da Conceição, E., Rezende, M., Fiuza, T. de S., et al. (2016). Essential oil composition, antimicrobial and pharmacological activities of Lippia sidoides cham. (verbanaceae) from São Gonçalo do Abaeté, Minas Gerais, Brazil. Pharmacognosy Magazine, 12(48), 262-270. http://www.phcog.com/article.asp?issn=0973-1296

Mustafa, G., Arif, R., Atta, A., Sharif, S., \& Jamil, A. (2017). Contents List available at RAZI Publishing Bioactive Compounds from Medicinal Plants and Their Importance in Drug Discovery in Pakistan Matrix Science Pharma ( MSP ). Matrix Science Pharma, 1(1), 17-26.

Nader, T. T., Coppede, J. S., Amaral, L. A., Facchin, A. L., Pereira, A. M. S., \& Ferreira, L. M. (2010). Avaliação in Vitro Da Eficácia De Extratos De Plantas Medicinais Do Cerrado Frente Staphylococcus Aureus Isolado De Diferentes Fontes De Propriedades Leiteiras. Arquivos do Instituto Biológico, 77(3), 429433.

Nagoor Meeran, M. F., Javed, H., Al Taee, H., Azimullah, S., \& Ojha, S. K. (2017). Pharmacological Properties and Molecular Mechanisms of Thymol: Prospects for Its Therapeutic Potential and Pharmaceutical Development. Frontiers in pharmacology, 8, 380.

Namdari, H., Izad, M., Rezaei, F., \& Amirghofran, Z. (2019). Thymol as a reciprocal regulator of T cell differentiation: Promotion of regulatory T cells and suppression of Th1/Th17 cells. International immunopharmacology, 67, 417-426. Netherlands.

Oliveira, B. I. M. de, Cunha, L. S., Braga, L. L., Lima, A., \& Caixeta, L. D. (2018). Fitoterapia Na Odontologia : Levantamento Dos Principais. Psicologia e Saude em Debate, 4(1), 71-71.

Oliveira, D., Leitao, G., Bizzo, H., Lopes, D., Alviano, D., Alviano, C., \& Leitão, S. (2007). Chemical and Antimicrobial analyses of essential oil of Lippia origanoides H.B.K. Food Chemistry, 101, 236-240.

Pereira, S. L. da S., Praxedes, Y. C. M., Bastos, T. C., Alencar, P. N. B., \& da Costa, F. N. (2013). Clinical effect of a gel containing Lippia sidoides on plaque and gingivitis control. European Journal of Dentistry, 7(1), 28-34.

Petrovska, B. B. (2012). Historical review of medicinal plants' usage. Pharmacognosy Reviews, 6(11), 1-5.

Rattanachaikunsopon, P., \& Phumkhachorn, P. (2010). Assessment of factors influencing antimicrobial activity of carvacrol and cymene against Vibrio cholerae in food. Journal of Bioscience and Bioengineering, 110(5), 614-619. Japan.

Rodrigues, I. S. C., Oliveira, D. B. de, Menezes, P. C. B. de, Costa, F. N. da, Carlos, M. X., \& Pereira, S. L. da S. (2013). Effect of Lippia sidoides in mouthrinses on de novo plaque formation: A double-blind clinical study in humans. Indian Journal of Dental Research, 24(5), 533-536.

Rosa, C. da, Câmara, S. G., \& Béria, J. U. (2011). Representações e intenção de uso da fitoterapia na atenção básica à saúde. Ciência \& Saúde Coletiva, 16(1), $311-318$.

Santos, R. L., Guimaraes, G. P., Nobre, M. S. C., \& Portela, A. S. (2011). Análise sobre a fitoterapia como prática integrativa no Sistema Único de Saúde. Revista Brasileira de Plantas Medicinais, 13(4), 486-491.

Saúde, M. da. (2018). Plantas Medicinais Brasileiras de interesse ao SUS. 
Sen, S., \& Chakraborty, R. (2016). Revival, modernization and integration of Indian traditional herbal medicine in clinical practice: Importance, challenges and future. Journal of traditional and complementary medicine, 7(2), 234-244. Elsevier. https://pubmed.ncbi.nlm.nih.gov/28417092

Shakya, A. K. (2016). Medicinal plants: Future source of new drugs. International Journal of Herbal Medicine, 4(4), 59-64.

Silva;, E. F. da, Vieira, T. J. N., \& Gurgel, G. C. D. de L. (2017). Atividade biológica "in vitro" do fitoconstituinte timol sobre espécies do gênero Candida. Revista de Pesquisa Interdisciplinar, 2(Setembro), 677-685.

Singh, A., Verma, R., Murari, A., \& Agrawal, A. (2014). Oral candidiasis: An overview. Journal of oral and maxillofacial pathology: JOMFP, 18(Suppl 1), S81-5.

Soares, B. V., \& Tavares-Dias, M. (2013). Espécies de Lippia (Verbenaceae), seu Potencial Bioativo e Importância na Medicina Veterinária e Aquicultura. Biota Amazônia, 3(1), 109-123.

Souza, Z. N. de, Barros, B. R. da S., Silva, K. S. da, Silva, R. S. da, \& Melo, C. M. L. de. (2020). Plantas Medicinais Utilizadas No Nordeste Do Brasil: Uma Revisão De Literatura. Ciências Da Saúde E Suas Contribuições Sociais, 115-132.

Tortora, G. J., \& Derrickson, B. (2016). Princípios de Anatomia e Fisiologia (14/20167.). Guanabara Koogan.

Vázquez-Sánchez, D., Galvão, J. A., Mazine, M. R., Gloria, E. M., \& Oetterer, M. (2018). Control of Staphylococcus aureus biofilms by the application of single and combined treatments based in plant essential oils. International journal of food microbiology, 286, 128-138. Netherlands.

van der Velden, A. W., Sessa, A., Altiner, A., Pignatari, A. C. C., \& Shephard, A. (2020). <p>Patients with Sore Throat: A Survey of Self-Management and Healthcare-Seeking Behavior in 13 Countries Worldwide</p >. Pragmatic and Observational Research, Volume 11, 91-102.

Veras, H. N.H., Rodrigues, F. F. G., Botelho, M. A., Menezes, I. R. A., Coutinho, H. D. M., \& Da Costa, J. G. M. (2014). Antimicrobial effect of lippia sidoides and thymol on enterococcus faecalis biofilm of the bacterium isolated from root canals. (A. I. Vela \& J. Yoon, Eds.)The Scientific World Journal, 2014, 471580. Hindawi Publishing Corporation. https://doi.org/10.1155/2014/471580

Veras, Helenicy N.H., Rodrigues, F. F. G., Botelho, M. A., Menezes, I. R. A., Coutinho, H. D. M., \& Costa, J. G. M. (2017). Enhancement of aminoglycosides and $\beta$-lactams antibiotic activity by essential oil of Lippia sidoides Cham. and the Thymol. Arabian Journal of Chemistry, 10, S2790-S2795.

Vicente, V. A., Polleto, M. M., Neiva, I. F., Pinto, J. V. T., Braga, S. F., Moreira, M., \& Lavoranti, O. J. (2010). Relação entre a prevalência da doença cárie e risco microbiológico. Brazilian Dental Science, 11(2).

WHO Report. (2019). WHO Global report on traditional and complementary medicine 2019. World Health Organization. Geneva PP. World Health Organization. https://apps.who.int/iris/bitstream/handle/10665/312342/9789241515436-eng.pdf?ua=1

World Health Organization. (2020). Considerations for the provision of essential oral health services in the context of COVID-19. Interim Guidance (Vol. 3 August). Geneva PP - Geneva: World Health Organization. https://apps.who.int/iris/handle/10665/333625

Yang, T.-S., Chao, L. K.-P., \& Liu, T.-T. (2014). Antimicrobial activity of the essential oil of Glossogyne tenuifolia against selected pathogens. Journal of the science of food and agriculture, 94(14), 2965-2971. England.

Yeh, C.-F., Li, W.-Y., \& Hsu, Y.-B. (2014). Klebsiella pneumoniae pharyngitis mimicking malignancy: a diagnostic dilemma. Infection, 42(6), 1047-1050.

Yousef, R. Y., \& Yousef, R. Y. (2010). Comparison of The Bacteriology of Tonsil Surface and Core in Bacterial Profile Isolated from Children with Chronic Tonsillitis. Medical Journal of Babylon, 7(1), 52-57.

Yudice, E., Col, R., de Mattos, E., dos Santos Menezes Gaiotto Daros, V., Farias, F., Guilherme, M., Vieira, E., et al. (2018). O Uso de Prebiótico e Probiótico no Tratamento da Obesidade. XXI I Congresso Brasileiro de Nutrologia, 11, 14-21. 